JOURNAL DE PHYSIQUE IV

Colloque C4, supplément au Journal de Physique III, Volume 5, mai 1995

\title{
Spontaneous Localization of Vibrational Energy
}

\author{
D.W. Brown and L. Bernstein* \\ Institute for Nonlinear Science, University of California, San Diego La Jolla, CA 92093-0402, U.S.A. \\ * Department of Mathematics, Box 8085, Idaho State University, Pocatello, ID 83209-8085, U.S.A.
}

\begin{abstract}
In this paper we illustrate a number of the characteristic manifestations of soft anharmonicity in the behavior of lattice vibrations, both in and out of thermal equilibrium. In particular, we focus on various ways in which vibrational energy may come to be localized as a consequence of anharmonicity, even in defect-free lattices. These include the "overpopulation" of anharmonic vibrations in thermal equilibrium, the inhibition of dispersion, and the enhancement of spatial coherence. The relevance of the spontaneous localization of vibrational energy in the formation of "hot spots" is discussed, with particular emphasis on the unstable evolution of high-amplitude initial conditions as may be found in initiating shocks.
\end{abstract}

\section{Research is what I'm doing when I don't know what l'm doing. -- Werner von Braun}

\section{INTRODUCTION}

The process of detonation is remarkable in part because of its strong linkage of microscopic events and macroscopic dynamical effects. This cascade across space and time scales has as one of its consequences that no one scientific discipline embraces the phenomenon in its entirety. This paper is addressed to the anharmonic behavior of crystal vibrations in the initiation phase of detonation, defined as the interval between the delivery of a low-amplitude shock into virgin material and the later development of a steady-state detonation front. The phenomenon of greatest interest here is the localization of vibrational energy, particularly whether such localization may facilitate the initiation of chemistry that may ultimately support detonation. While it is possible that such localization may also be involved in the steady-state propagation of the detonation front, the strong role of chemistry in the steady-state problem suggests at least that the space-time interval in which anharmonic vibrational effects may be distinguishable as such may be strongly reduced relative to the initiation phase.

In a general sense, the subject to be developed here may be associated with the established concept of "hot spots"; however, it is important to emphasize an essential distinction between the usual conception of hot spots and that of anharmonic localization. While many implementations of the idea could be enumerated, the established concept of hot spots is almost universally associated with material defects that become superheated when stressed by an impinging shock. Though explosive sensitivity is strongly influenced by inhomogeneities of diverse origins, crystalline or nearly defect-free energetic materials are also found to be detonable, albeit at elevated shock pressures. This suggests that even in homogeneous materials processes exist that may be capable of achieving significant energy localization under the influence of a sufficiently energetic drive. This is precisely the nature of soft anharmonic vibrations.

A vibration may be characterized as soft (hard) if the vibrational frequency decreases (increases) with increasing amplitude. In quantum-mechanical terms, a soft (hard) vibration is one whose energy level spacing decreases (increases) with increasing vibrational quantum number. These characterizations apply 
equally to symmetric potentials as we use in this paper, or to asymmetric potentials such the Morse or Lennard-Jones [1]. In the case of vibrations whose potentials are symmetric with respect to the equilibrium amplitude, a soft (hard) vibration is one whose potential increases more slowly (rapidly) with increasing amplitude than does the harmonic reference potential describing small oscillations in the same oscillator. These characterizations may change as a function of amplitude; consider, for example, an oscillator whose potential first increases more slowly than harmonic, but which later increases more rapidly than harmonic. For the smooth potentials characteristic of mechanical equilibria, such changes the character of the anharmonicity occur only above some finite amplitude, such that a vibration may be characterized uniquely as soft or hard depending on the leading anharmonic correction at low amplitudes; it is this characterization that we apply throughout this paper.

\section{THERMODYNAMICS}

It will be useful in the following to consider the anharmonic oscillator described by the Hamiltonian

$$
H=\frac{1}{2} \dot{x}^{2}+\frac{1}{2} x^{2}-\frac{\varepsilon}{4} x^{4}
$$

for soft anharmonicity, $\varepsilon>0$. (It is well to note that this oscillator is thermodynamically unstable, since the potential is unbounded below as $|x|$ increases beyond $x_{c}=\varepsilon^{-1 / 2}$, and should be handled with care when used in more than a qualitative sense.)

The equipartition theorem in its general form [2] reads

$$
<\dot{x} \frac{\partial H}{\partial \dot{x}}>=<x \frac{\partial H}{\partial x}>=k_{B} T .
$$

Using the anharmonic oscillator Hamiltonian (1), this yields

$$
<H>=k_{B} T+\frac{\varepsilon}{4}\left\langle x^{4}\right\rangle,
$$

which indicates that the equilibrium expectation value of the total energy of a soft anharmonic oscillator exceeds that of a harmonic oscillator. This qualitative conclusion does not depend on the frequency of the harmonic oscillator used for comparison, nor on the degree of freedom underlying the vibration, since all harmonic oscillators in equilibrium at the same temperature share the same energy expectation value. Consequently, we may conclude that any soft anharmonic oscillator will equilibrate to an average energy higher than that of any harmonic oscillator at the same temperature.

The magnitude of this excess is not necessarily large, of course. Clearly, the magnitude of this disparity increases with the strength of the anharmonicity, but for the same value of the anharmonicity (e.g., the same $\varepsilon$ ), the expected value of the higher moments (e.g. $\left\langle x^{4}\right\rangle$ ) will be larger in oscillators having lower frequencies at low amplitude (see below). This suggests that we may expect anharmonicity-driven concentrations of energy to be most pronounced among the lowest-lying vibrational modes of a complex system.

It is well to note that because the principal effect of an impinging shock is to promptly compress material passing under it, energy is first delivered into low-lying vibrational modes associated with volumetric compression; these include the acoustic modes associated with translations of the center of mass of a unit cell, librational modes associated with rigid-body rotations of principal unit cell constituents, and the lowest-lying optical modes associated with relative translations of unit cell constituents. The remaining modes of vibration are associated more essentially with deformations of individual molecules, and being generally substantially higher in frequency, couple less effectively with shocks. (For cautionary observations in this regard, see e.g. Ref. [3].)

Sufficiently far behind the shock, we may assume the material to come to equilibrium at a temperature substantially higher than that of the unshocked material. Under such conditions, the intrinsic anharmonicity of any vibration should be more pronounced, with the strongest anharmonic effects evident in the 
low-lying vibrations.

In the following, we make use of the notion of a "reference oscillator", by which we mean a hypothetical harmonic oscillator in the same degree of freedom as the anharmonic oscillator in question, such that the frequency of this harmonic oscillator matches the frequency of the anharmonic oscillator at small amplitudes. This allows us to focus on the manner in which the properties of anharmonic oscillators deviate from the harmonic ideal. In such comparisons, quantities associated with anharmonic oscillators are decorated with tildes (e.g., $\tilde{V}$ ), while harmonic reference quantities are decorated with zeros (e.g., $V_{0}$ ) to indicate that the anharmonicity has been set to zero. Moreover, for simplicity we explicitly consider only vibrational potentials symmetric about the equilibrium.

Since the potential of a soft anharmonic oscillator is less than or equal to that of the harmonic reference oscillator at all amplitudes $\left(\vec{V}(x) \leq V_{0}(x)\right)$, it is evident that the partition function $\bar{Q}$ of an anharmonic oscillator should be greater than the partition function $Q_{0}$ of the harmonic reference oscillator at all temperatures; i.e.,

$$
\tilde{Q}=\iint e^{-\left[1 / 2 p^{2}+\bar{V}(x)\right] / k_{B} T} d p d x \geq \iint e^{-\left[1 / 2 p^{2}+V_{0}(x)\right] / k_{B} T} d p d x=Q_{0} .
$$

Consequently, the free energy of the soft anharmonic oscillator

$$
A=E-T S=-k_{B} \ln Q
$$

is lower $\left(\tilde{A}<A_{0}\right)$, and (using the equipartition result) the entropy and specific heat higher $\left(\tilde{S}>S_{0}\right.$, $\tilde{C}_{v}>C_{v}^{0}$ ) than in the harmonic reference oscillator.

From such considerations we may draw some conclusions regarding energy fluctuations. The variance of the total energy in thermal equilibrium is related to the specific heat by the relation

$$
<\Delta E^{2}>=k_{B} T^{2} C_{v} .
$$

In view of the equipartition theorem, we may conclude that the normalized variance of the total energy of a single harmonic oscillator at thermal equilibrium is equal to unity

$$
\frac{\left\langle\Delta E_{0}^{2}\right\rangle}{\left\langle E_{0}\right\rangle^{2}}=1 \text {. }
$$

On the other hand, the normalized energy variance for our anharmonic oscillator is given by

$$
\frac{\left\langle\Delta \tilde{E}^{2}\right\rangle}{\langle\tilde{E}\rangle^{2}}=\left[1+\frac{\varepsilon}{4 k_{B}} \frac{\partial\left\langle x^{4}\right\rangle}{\partial T}\right]\left[1+\frac{\varepsilon}{4 k_{B}} \frac{\left\langle x^{4}\right\rangle}{T}\right]^{-2}>1 .
$$

Expanding the partition function in orders of the anharmonicity parameter $\varepsilon$, we may compute $\left\langle x^{4}\right\rangle$ approximately as

$$
\begin{aligned}
\left.<x^{4}\right\rangle & =\left\langle x^{4}>_{0}+\frac{\varepsilon}{4 k_{B} T}\left[\left\langlex^{8}>_{0}-\left\langle x^{4}>_{0}^{2}\right]+O\left\{\varepsilon^{2}\right\}\right.\right.\right. \\
& \approx 3\left(k_{B} T\right)^{2}+24 \varepsilon\left(k_{B} T\right)^{3}+O\left\{\varepsilon^{2}\right\}
\end{aligned}
$$

in which the zero subscript indicates the thermal expectation value in the absence of anharmonicity. From this one may show that the normalized energy variance is greater than unity in the soft anharmonic case, indicating that relative to harmonic oscillators, the energy fluctuations of soft anharmonic oscillators are marked by "higher highs" and "lower lows", or a relative preponderance of high energy fluctuations.

\section{DUTY CYCLE AND DISSIPATION}

The duty cycle of an oscillation is the ratio of the fractions of a cycle that the oscillator spends in two essentially exclusive states; for example, a square wave may be "on" for one third of a cycle and "off" for 
two thirds, for a duty cycle of 1:2. For our purposes, it is useful to consider as exclusive states the conditions that the energy of an oscillation reside a) primarily in the kinetic form, or b) primarily in the potential form. For harmonic oscillators, the energy spends equal times in the two forms, for a duty cycle of 1:1. For soft anharmonic oscillators, the period of oscillation is greater than that of the reference harmonic oscillator, and most of this increase is accounted for by a lengthening of the time that the energy spends in the potential form. This leads to a duty cycle of $1: 1+\eta$, where $\eta$ is a measure of the lengthening of the period due to anharmonicity.

The duty cycle affords a dynamical understanding of our previously-obtained equipartition result. Since the kinetic energy of both harmonic and anharmonic oscillators is a quadratic degree of freedom, the equipartition theorem requires that both harmonic and anharmonic oscillators have the same average kinetic energy. However, since the energy of soft anharmonic oscillators spends relatively more time in the potential form than is the case for harmonic oscillators, the equality of the average kinetic energies forces an inequality of the average total energies such that the greater average energy is found in the soft anharmonic oscillator.

The duty cycle also allows some conclusions to be drawn regarding dissipation. Using the Langevin approach, one may model the dynamics of an oscillator in contact with a heat bath by the equation

$$
\ddot{x}=-\frac{\partial V}{\partial x}-\gamma \dot{x}+f(t)
$$

in which $\gamma$ represents the dissipation and $f(t)$ is a zero-centered stochastic force. This implies that energy drains from the oscillator according to

$$
\dot{E}=-2 \gamma(\text { Kinetic Energy })+\dot{x} f(t) \text {. }
$$

Thus, in the case of a freely decaying oscillation $(f(t) \approx 0)$, the energy drains nonuniformly in time, and primarily during that fraction of the cycle in which the energy resides primarily in the kinetic form. Since soft anharmonicity extends the time that an oscillator's energy resides in the potential form, we may conclude that softened oscillations decay more slowly than corresponding harmonic oscillations. Since the low amplitude oscillations of a soft oscillator are quite harmonic, we may conclude as well that low amplitude oscillations of a soft oscillator decay more rapidly than do high amplitude oscillations in the same oscillator. This suggests a relative preponderance of high energy fluctuations in soft oscillators, consistent with the thermodynamic computation of the energy variance in the preceding section.

All of the foregoing considerations point to a notion of "statistical localization"; that is, that there exists a thermodynamic gradient for concentrating energy in anharmonic vibrations. This concentration may occur spatially if, for example, an anharmonic impurity is embedded in a lattice of harmonic oscillators. Or, in the absence of impurities it may create disparities between classes of modes if, say, librational modes are rich in anharmonicities while acoustic modes (say) are not. Even in our case study of a single soft anharmonic oscillator, we find a tendency for high-energy fluctuations to be damped less vigorously than low-energy fluctuations, resulting in a relative persistence of high-energy fluctuations, allowing one to think of energy localization as occurring in time [4].

\section{SPATIAL COHERENCE AND THE SOLITON CONCEPT}

The localization of energy in time, that is, the anomalous frequency and/or persistence of high energy states, is one factor in promoting the initiation of chemistry as in the hot spot concept; another important factor, however, is the localization of energy in space. The kind of localization discussed to this point could occur in a meaningful way without exhibiting any spatial coherence whatever; e.g., two oscillators that in a random interval of time happen to undergo similar high energy fluctuations might be nearest neighbors or might be widely separated in space. Particularly in energetic materials, quite different consequences might be expected to result if high energy fluctuations were randomly distributed in space or were correlated so as to occur preferentially in a more limited domain.

While there are many ways that energy concentrations may be achieved in the neighborhood of defects, 
particularly in non-equilibrium situations such as as the initiation phase of detonation [5,6], we focus here on mechanisms that may support the maintenance of spatial coherence in homogeneous materials.

The most elementary interaction between neighboring oscillators is the harmonic one, as in

$$
H\{\dot{x}, x\}=\sum_{n}\left[\frac{1}{2} \dot{x}_{n}^{2}+\frac{c^{2}}{l^{2}}\left(x_{n}-x_{n-1}\right)^{2}+V\left(x_{n}\right)\right],
$$

giving rise the propagation of energy through space. In absence of a potential $V(x)$, this represents acoustic vibrations in which $c$ is the speed of sound. The effect of the nearest-neighbor interaction is to drive neighboring oscillators toward the same phase, and thus to establish coherence on the smallest spatial scale. Dynamically, this local drive toward spatial coherence has the effect of dispersing any localized energy distribution, since such a distribution constitutes a deviation from perfect alignment. Thus, the main dynamical consequence of building spatial coherence in vibrational systems is dispersion.

Thus we have the tendency of soft anharmonicity to concentrate energy without regard to spatial coherence, and the tendency of spatial coherence to lead to the dispersion of energy. Since these opposing tendencies coexist in the same dynamical system, it is reasonable to expect optimal solutions to achieve some degree of balance between them. Indeed this is the case, and it is this physical need to balance anharmonic focusing against dispersion that gives rise to the concept of solitary waves, and their idealization in the concept of the soliton [7-11]. The relevance of the concept does not depend on the validity of the ideal, however. Figure 1 shows harmonic and anharmonic systems initialized with the same Gaussian pulse in their initial amplitudes. The pulse in the harmonic chain disperses quickly, reaching the (periodic) boundary in just a few frames of this particular "movie". On the other hand, the pulse in the anharmonic chain resists dispersion, retaining its integrity many times longer than in the harmonic case. Though the parameters for this illustration were chosen to make an interesting presentation, it should be noted that nothing about this illustration approaches the soliton ideal; the Gaussian initial condition does not correspond to any soliton solution, the relatively short, discrete chain does not approximate the continuum or infinite-size scenarios typical of soliton systems, and the "leakage" apparent in this solution, albeit small and slow, reflects the non-ideality of the represented phenomenon [12-15].

It is worth noting that the slowly-decaying localized excitation in Figure 1 undergoes a kind of "breathing" motion suggestive of internal modes. This is a general characteristic of soliton-like states that is related to their enhanced lifetimes and dynamical stability; within limits, such anharmonic excitations respond flexibly to perturbations, transiently deforming during interactions with other excitations or defects such that the integrity of the soliton-like state is approximately preserved.

\section{PHONONS VS. SOLITONS}

"Phonon", as commonly used, denotes a quantum of vibrational energy in any of the normal modes of vibration of an extended medium. ("Phonon" is sometimes reserved for acoustic phonons, and other terms such as "libron" and "vibron" used to denote the quanta of other classes of vibration, but we ignore such distinctions here.) Implicit, however, are a few additional qualifiers; for example, though in principle normal modes can be found for defective crystals, one often restricts "phonon" to the normal modes of perfect crystals, and describes defect effects in terms of phonon-phonon scattering mediated by defects. Moreover, "normal modes" usually implies the harmonic approximation, leaving anharmonic effects to be described in terms of multi-phonon scattering and the finite lifetimes for phonons this implies [5,16,17].

"Solitons" may be viewed as the particle-like subset of the nonlinear normal modes of an extended anharmonic system, usually in a classical or semiclassical approximation. Again, some qualifiers are in order. The notion of nonlinear normal modes is exact only for completely integrable systems, and relatively few anharmonic systems of practical interest are completely integrable. Nonetheless, it can be meaningful to regard a practical system as being "nearly" integrable, and to characterize its non-integrability through "scattering" interactions between the nonlinear normal modes (including solitons) of the nearby integrable system, leading to imperfect soliton behaviors including finite soliton lifetimes [18-20]. 


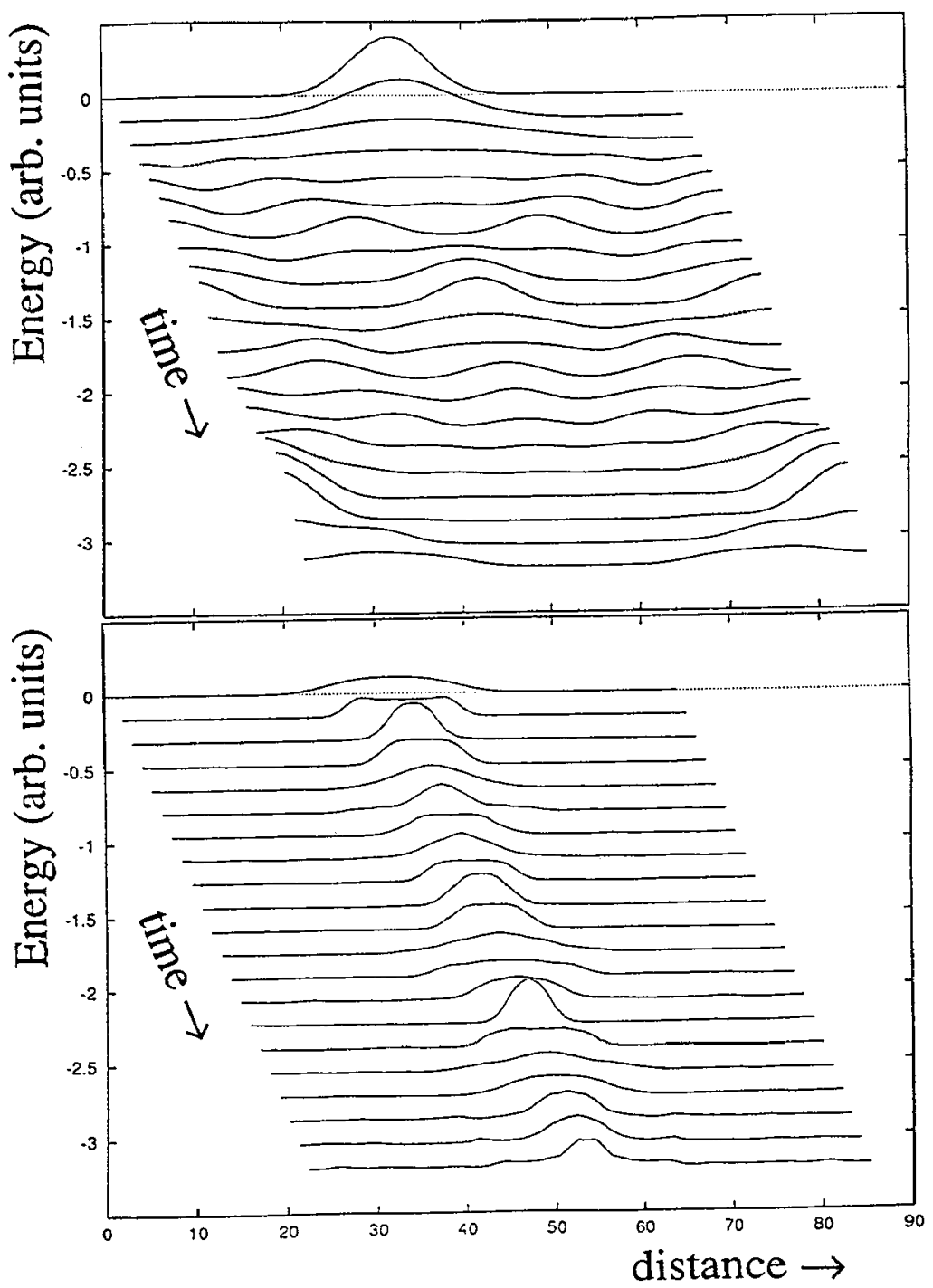

Figure 1. Evolution of identical Gaussian initial conditions in the oscillator amplitudes at zero temperature. Upper panel, harmonic; lower panel, anharmonic. Note that the initial energy profile is different in the two cases; this is because the initial energy is entirely in the potential form, which for a given oscillator amplitude is lower in the anharmonic system than in the harmonic system.

Since most practical scenarios are not perfect fits to either the phonon or soliton picture, it is well to understand what is to be gained by using the generally more-complicated soliton picture. The essential limitation of the phonon picture is that accounting for anharmonic effects by multi-phonon scattering tends to lose information relating to the phonon-phonon coherences such as are involved in the localization of vibrational energy. In the soliton picture, the anharmonicity may be viewed as being separated into a part associated with a "nearby" completely integrable soliton-supporting system and the remainder of the anharmonicity treated as a perturbation, though in a manner that is generally much more complex than in the phonon picture. The advantages are that the soliton picture is well-suited to the description of coherence effects, and the size of the remaining scattering perturbation is smaller than in the phonon picture. 


\section{THERMAL EQUILIBRIUM SIMULATION}

For simulating anharmonic lattices at finite temperature, we need to choose an anharmonic potential that does not suffer the instabilities noted in Eqn. (1). For simplicity, we choose a symmetric potential having a unit frequency at small amplitude, but that saturates monotonically to a constant as $x \rightarrow \pm \infty$

$$
V(x)=\frac{x^{2}}{2+\varepsilon x^{2}}=\frac{1}{2} x^{2}-\frac{\varepsilon}{4} x^{4}+O\left\{\varepsilon^{2}\right\} .
$$

(The first two terms in the Taylor series expansion of this potential are the same as in Eqn. (1).) The system is otherwise as indicated in Eqn. (12), and is simulated using Langevin terms as in Eqn. (10), following Heun's method [21,22]. Each simulation begins with a warm-up phase, during which the system is warmed to the desired temperature from a zero-temperature initial state. Measurement begins only after thermal equilibrium diagnostics fall within required tolerances.

The results of such simulation are quite different from the zero-temperature example of Figure 1. Typical out-takes from thermal equilibrium simulations of both harmonic and anharmonic systems are shown in Figure 2. The outstanding characteristic of these simulations is that the difference between the harmonic and anharmonic simulations is not dramatic; it is difficult with the naked eye and a common vocabulary to characterize the differences in texture. One may say that the energy density in the anharmonic simulation is generally more smooth, and marked by a smaller number of high energy excursions that are generally more broad in space; i.e., the anharmonic simulation displays more spatial coherence.

We can be more objective in our characterization by constructing an energy correlation function:

$$
C_{n}=\left[N \underset{m}{\sum} E_{m} E_{m+n}>\right]\left(<\sum_{m} E_{m}>\right]^{-2}, \quad \quad \sum C_{n}=N .
$$

In the absence of any spatial coherence, this should consist of a central spike above a uniform background

$$
C_{n}=\delta_{n 0} \frac{N\left(C_{0}-1\right)}{N-1}+\frac{N-C_{0}}{(N-1)} .
$$

Any deviation from this form indicates the existence of spatial coherence. The result of computing this correlator for the two scenarios of Figure 2 are shown in Figure 3. The result of this computation affirms quantitatively that soft anharmonicity is responsible for an increased spatial coherence in the fluctuating energy density typical of thermal equilibrium.

The contrast between the strong effects shown in Figure 1 and the weak effects shown in Figures 2 and 3 contains and important message. It is often possible to construct long-lived localized excitations in the zero-temperature limit of systems of soft anharmonic oscillators; these excitations tend to decay rapidly at elevated temperatures, however, because strong spatial coherence generally exacts a high cost in entropy that at finite temperatures strongly suppresses the probability of their occurrence.

\section{RESPONSE TO SHOCK}

The nature of the microscopic response of materials to the passage of a shock front is known only in a rough sense, and primarily in terms of thermodynamic constraints. The nature of the dynamic response on the molecular scale is essentially unknown, and so it is necessary to work from reasonable hypotheses $[3,23,24]$. To the extent that the shock front may be considered to be "sharp", it would appear reasonable to expect the shock to deliver an impulse to at least the low-lying vibrations associated with the unit cell being traversed. The passage of a shock through a finite piece of material would therefore involve the delivery of a sequence of impulses on the molecular scale. The "initial condition" this provides for the vibrational system consists of a super-thermal energy density, which may, however, be modulated on a length scale related to the sequence of phase delays arising from the finite time elapsing between the delivery of impulses to successive unit cells.

Uniform waves in systems with soft anharmonicities are characteristically vulnerable to modulational 


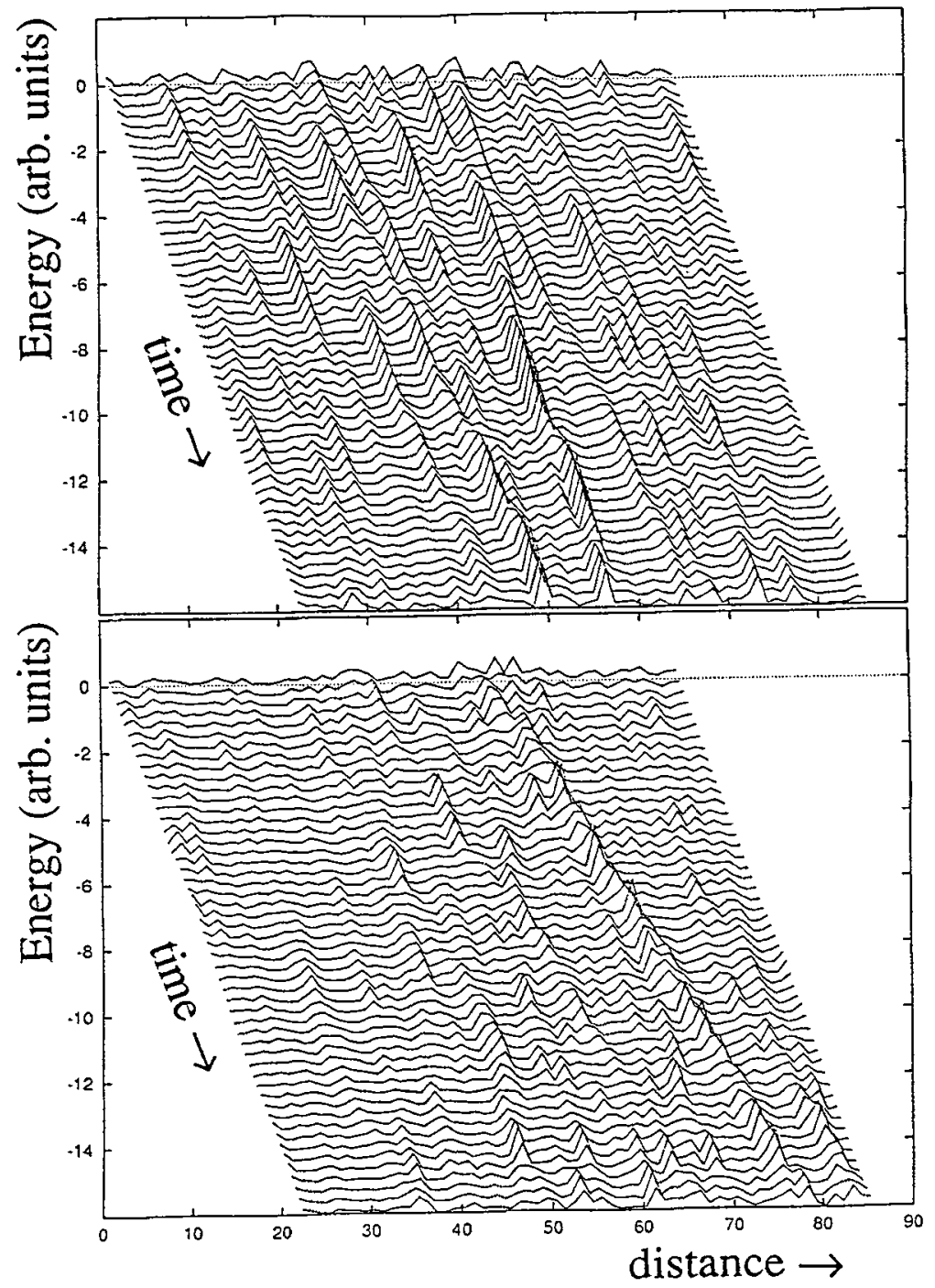

Figure 2. Energy densities at finite temperature. Upper panel, harmonic; lower panel, anharmonic.

instabilities; that is, a monochromatic wave might exist indefinitely, but with the addition of a small spatially-periodic perturbation creating a weak modulation of the fundamental wave, the system may evolve toward a very different state $[9,10,15]$. Depending on the particular circumstances, monochromatic waves might be unstable to perturbations of any wavelength or only in a limited range (e.g., long wavelengths), or may have an amplitude threshold, and stability may depend on characteristics of the monochromatic wave being perturbed. Characteristically, however, the destabilized wave evolves toward a pulse-train waveform in which the pulses approximate solitary waves of the underlying or "nearby" nonlinear wave equation (if such exist). The rate at which perturbations grow typically depends on both the strength of the soft anharmonicity and the amplitude of the fundamental wave, such that perturbations grow much more rapidly in softer modes driven to higher amplitudes. Thus, it would be the more intense shocks that would be more likely to cause initial perturbations to grow, and, recalling earlier discussion, this growth would be more likely to be achieved in the low-frequency modes that couple well 


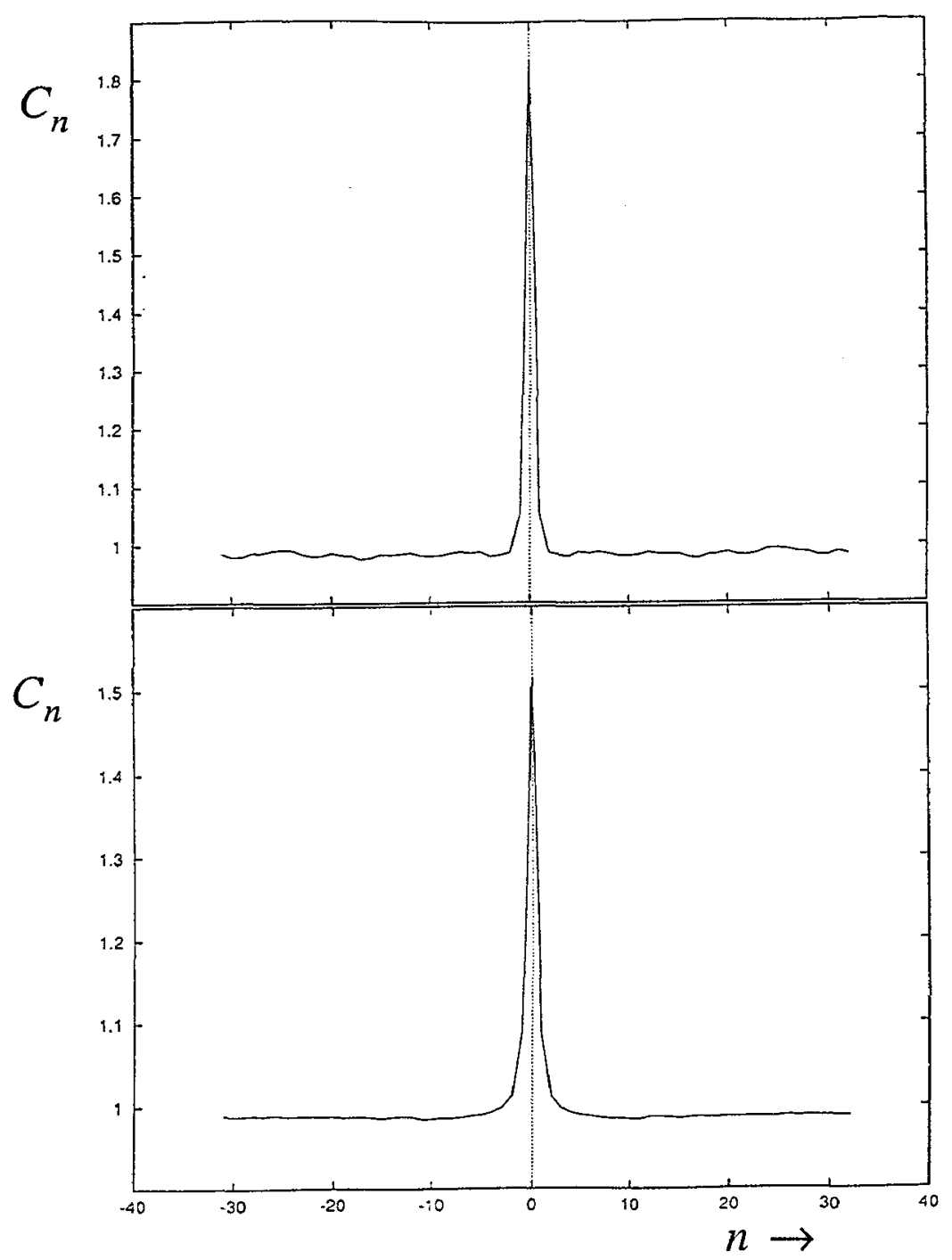

Figure 3. Energy-energy correlation function in site space according to Eqn. (14). Upper panel, result of thermal equilibrium simulation in the harmonic system. Lower panel, result of thermal equilibrium simulation in the anharmonic system at the same temperature. Correlations in the harmonic system are indiscemible from the background beyond the first neighbor; in the anharmonic system, correlations are discemible above background out to fifth neighbors.

with the shock.

In Figure 4 we illustrate the result of initializing chains of oscillators with nearly-uniform initial energy distributions upon which are imposed weak spatially-periodic modulations. The harmonic chain disperses the perturbation as it would any other nonuniformity in amplitude. On the other hand, the modulation in the anharmonic chain grows in time, transforming the original nearly-uniform energy distribution into a train of pulses, each of which spans a number of unit cells within which a several-fold amplification of the original energy density is achieved for a nontrivial interval of time. This could be viewed as local heating, but actually much more coherent, achieving local deformations that much more likely than thermal fluctuations to interact with electronic excitations and perhaps assist in the initiation of chemsitry $[25,26]$ 


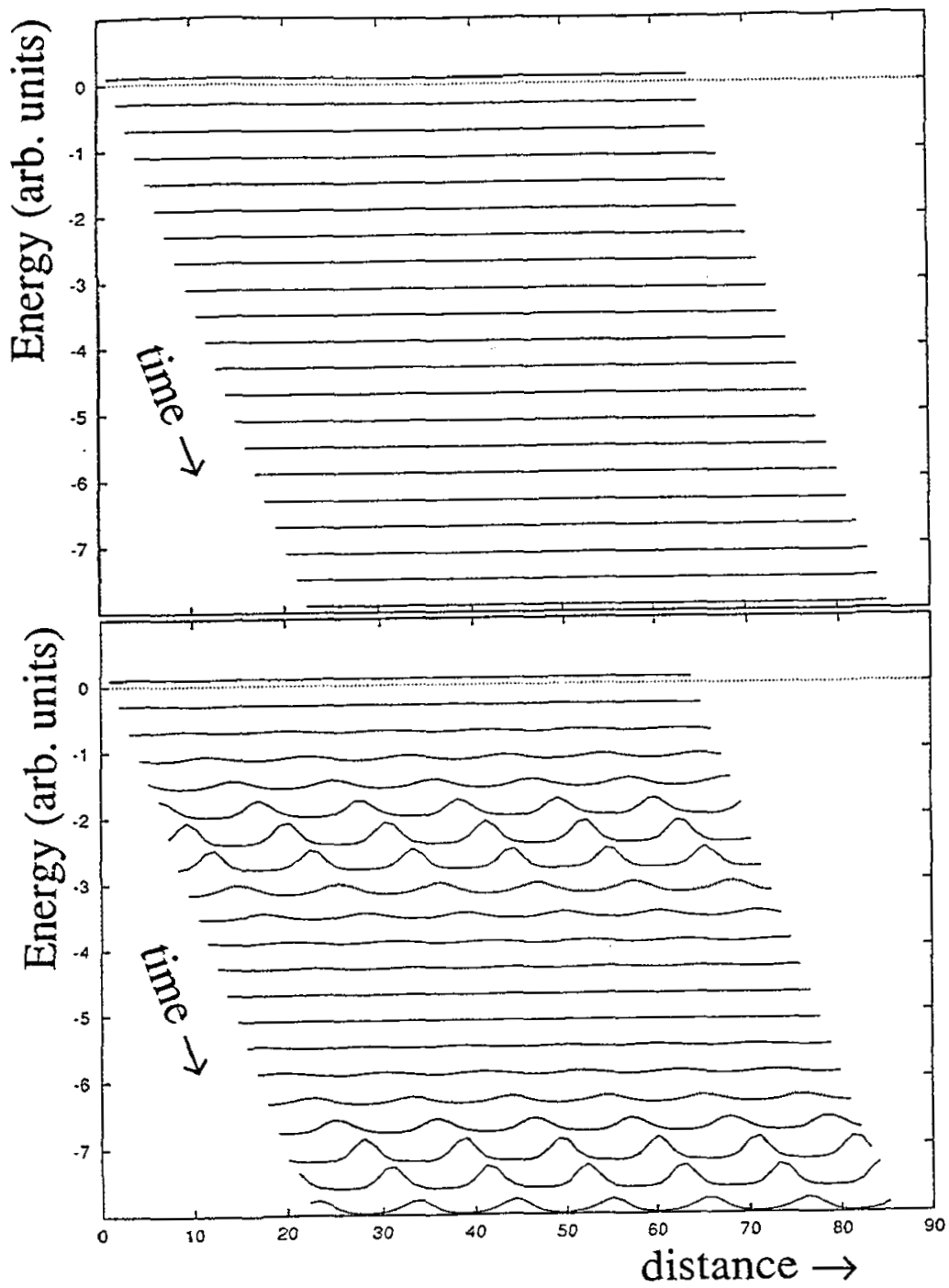

Figure 4. Energy density profile for non-uniform non-equilibrium excitation. Upper panel, harmonic system; lower panel, anharmonic system. The weak sinusoidal perturbation does not grow in the harmonic system, while in the anharmonic system the perturbation is amplified, resulting in transient energy focusing.

This dramatic focusing is a transient phenomenon that is eventually degraded as the shocked material equilibrates to a new local temperanure. Anharmonic effects as discussed in previous sections should be more pronounced in the new thermal equilibrium established behind the shock, but cannot be expected to be as dramatic as in the initial transient.

\section{CONCLUSION}

In this paper we have sketched a number of the characteristic manifestations of soft anharmonicity in systems of oscillators. We have seen how isolated soft anharmonic oscillators at finite temperature have mean energies higher than would be expected for harmonic oscillators, and are distinguished by energy fluctuations that tend to be longer-lived at higher energies than their hamonic counterparts. These characteristics motivate a notion of "statistical localization," the root of which is the entropic drive to 
sample the extra phase space rendered accessible by the softening of the vibrational potential.

At zero temperature, entropic effects are frozen out, laying bare the purely dynamical consequences of anharmonicity. The fundamental dynamical phenomena are the tendencies of soft anharmonicity to focus energy and the tendency of harmonic transport terms to disperse it; it is the interplay of these tendencies that is the source of interesting behavior. In its purest form, the balancing of focusing and dispersion leads to the formation of robust particle-like excitations called solitons. Though for the most part solitons are idealizations of practical phenomena, the "nearness" of a practical system to an ideal soliton system tends to show up in "soliton-like" behaviors such as the anomalous persistence of localized excitations (See Figure 1.) or the transient formation of soliton-like pulses during the decay of high-amplitude initial data (See Figure 4).

Thermal equilibrium in vibrational systems is inhospitable to soliton-like excitations in the sense that it is typically difficult to resolve well-defined, long-lived vibrational excitations. The three-fold roots of this difficulty are that 1) the "substance" of which the excitation is made is thermal energy that is not conserved except in a statistical sense, being subject to loss through dissipation and being regenerated only stochastically, 2) solitons are low-entropy states that exact a significant free-energy cost at elevated temperatures, and 3 ) in order to achieve mean energies sufficiently high to allow a significant penetration of the anharmonic regime, the temperature must be significant as well, such that no thermally generated soliton can ever escape (1) and (2). Thus, soft anharmonic vibrations in thermal equilibrium execute a Sisyphean dance, perpetually driving thermal fluctuations toward a soliton-like order, only to see that growing order continuously degraded by never-ending waves of fluctuations. Consequently, solitonic effects show up not in the major attributes of thermal equilibria, but in the textures and shadings of an otherwise harmonic-looking picture (See Figures 2 and 3).

Of these various perspectives, the one most closely resembling the problem of initiation in energetic materials is that illustrated in Figure 4. It is well to realize, however, that the evolution of "hot spots" from weakly-structured but high-amplitude initial data is just a particularly dramatic manifestation of self-focusing; exactly the same mechanisms under thermal equilibrium conditions may appear much less provocative.

Such is the nature of nonlinearity.

\section{DISCUSSION}

\section{Q. Carl Melius}

Is there an upper limit in the space scales and/or time scales for solitons?

A. In principle no. The nonlinear Schrodinger equation, for example, has true infinite-lived soliton solutions of any width in space. In practice, however, the answer depends on parameters and how nonideal one's circumstance is. Lattice discreteness, for example, has a significant impact on would-be soliton behavior, with effects ranging from scattering to pinning to the total disintegration of localized initial states [12-15].

Q. Elaine Oran

How do you relate this work to that of Bob Wyatt (U. Texas, Austin) on oscillators coupled to a thermal bath? There was a study that tried to compare classical, semi-classical, and full quantum-mechanical results for this case. It seems that body of work is related to the first part of your lecture.

A. I am afraid I am unfamiliar with work to which you refer.

Q. Elaine Oran

What are your thoughts about "rogue waves" causing localization of energy enough to cause ignition in an energetic material?

A. My understanding of rogue waves is casual at best. Perhaps what is most important for us here is that these ship-eating waves that appear "out of nowhere" are not normal fluctuations of a fluid in thermal equilibrium, but arise from the interaction of ocean currents in certain parts of the world. Insofar as rogue 
waves reflect a driven instability of a medium, one may acknowledge some similarities, but I would be wary of pressing this analogy too far.

\section{Q. Paul Clavin}

Is it not possible that the shock change the thermodynamic picture from a double well to a single well?

A. Yes. This is an especially appealing possibility when considering the coupling of "internal" degrees of freedom (librational, vibronic, and molecular motions) with the translational degrees of freedom. It is natural in such considerations for the shape of the double well to be modulated by acoustic vibrations; in such cases the range of possible anharmonic effects is considerably increased. It is conceivable, for example, that a particularly large acoustic deformation might drive a double well to a single well form. But short of such a direct initiation of chemistry, it should be noted that the coupling of acoustic and other vibrations is typically itself a source of anharmonicity, as are the bonds supporting the acoustic waves themselves. (In fact, the latter can be credited for the modern discovery of solitons in the famous FermiPasta-Ulam problem [7].) Any one of these several sources of anharmonicity may be active in a particular process, or they may conspire to yield more pronounced effects.

\section{Q. Anatoly Dremin}

It is some prolongation of C. Melius' question. It is known that reaction originates under shock effect in liquid EM inhomogeneously (hot spots). My question is--how much time is necessary to generate hot spots by your mechanism in liquid EM?

A. I regret that owing to my unfamiliarity with liquids in general and energetic materials in particular, I cannot give a very satisfactory answer. Typically, however, there are multiple time scales involved in the formation and evolution of anharmonic excitations. In order for anharmonic excitations as we have discussed to be recognized as such, they must exhibit anomalous persistence relative to harmonic dynamics; therefore, the time scales for the harmonic dispersal of energy set lower bounds on the timescales for (soft) anharmonic motions. Having no meaningful numbers in hand for energetic materials, the actual numbers we used in Figure 4 were drawn from another problem of interest to us, the librational dynamics of molecular crystal 1-alanine. The librations of 1-alanine exhibit a very weak anharmonicity in a weakly dispersive mode, contributing in Figure 4 to a slow focusing over about 25 periods of a single harmonic reference oscillator; stronger anharmonicity, together with stronger dispersion and stronger drive would shorten this interval substantially. It should be noted perhaps that energy focusing can occur much more rapidly in three dimensions than in our example of a onedimensional chain since energy may be drawn from the transverse dimensions as well as from neighbors within the chain, and can result in much stronger focusing since anharmonicity in higher dimensions characteristically competes more effectively against dispersion [8].

\section{Q. Dick Miller}

On Monday, we heard J. Dick describe the interaction of a shock wave with a PETN single crystal in a particular orientation. He showed experimental evidence of plastic deformation coupled with a chemical possibly fluorescent effect. He then showed a crystallographic representation of the interaction of the shock with the molecules by a shearing mechanism in the lattice that was unperturbed by the shock. You showed similar interactions in the DNA example. How can this type of analysis help us to understand the chemical effects of the shock or the possibly electronically induced chemistry in this experiment?

A. The number of nonlinear effects in the vicinity of the shock front that might contribute to the promotion of chemistry is rather large: there might be local heating, as suggested by Figure 4, "catalysis" might occur as discussed in response to P. Clavin, the formation and movement of dislocations as emphasized by R. Armstrong [23], S. Coffey [24], and J. Dick [3] is certainly a nonlinear phenomenon, to name a few. An important area we have not addressed here is the (strong) interaction of electronic excitations with vibrations; in solid-state physics, this commonly goes by the name of the "polaron problem". In the polaron problem, one is generally concerned with the response of the material to the presence of the electronic excitation; under appropriate circumstances, the self-consistent state of the excitation and the lattice can be viewed as a soliton [12]. The problem you pose is to some extent the 
reverse: that is, rather than observing the response of a medium to the injection of an electron, you wish to observe the response of the electronic system to the injection of vibrational excitation. I would say that the theoretical apparatus of both problems should be quite similar, though one's approach would naturally be dictated by one's particular objectives. It should be noted perhaps that the Nobel prize in chemistry recently went to Rudolf Marcus, primarily for his extensive work on electron-transfer reactions, both between individual molecules and between molecules and interfaces such as electrodes; the methodology of this work overlaps considerably polaron theory. One can imagine, as suggested by the work of J. Dick, that the shock front creates a solid-solid interface at which electron transfer might occur in chemicallysignificant quantities if conditions of the Marcus-type analysis were to apply $[25,26]$.

\section{REFERENCES}

[1] T. Dauxois, M. Peyrard, and A. R. Bishop, Phys. Rev. E 47, 684-695 (1993); T. Dauxois, M. Peyrard, and A. R. Bishop, Phys. Rev. E 47, R44-R47 (1993); T. Dauxois, M. Peyrard, and A. R. Bishop, Physica D 66, 35-42 (1993); M. Peyrard, Acta Physica Polonica B 25, 955-992 (1994).

[2] Richard C. Tolman, The Principles of Statistical Mechanics (Oxford University Press, London, 1938).

[3] J. J. Dick, J. Appl. Phys. 70, 3572-3587 (1991); Appl. Phys. Lett. 60, 2494-2495 (1992); J. Phys. Chem. 97, 6193-6196 (1993); J. J. Dick and J. P. Ritchie, J. Appl. Phys. 76, 2726-2737 (1994).

[4] Lisa Bernstein, David W. Brown, and Katja Lindenberg, to be published.

[5] D. D. Dlott and M. D. Fayer, J. Chem. Phys. 92, 3798-3812 (1990); H. Kim, D. D. Dlott, J. Chem. Phys. 93, 1696-1709 (1990); D. D. Dlott, J. Opt. Soc. Am. B 7, 1638-1652 (1990); X. N. Wen, W. A. Tolbert, and D. D. Dlott, Chem. Phys. Lett. 192, 315-320 (1992); A. Tokmakoff, M. D. Fayer, and D. D. Dlott, J. Phys. Chem. 97, 1901-1913 (1993); X. N. Wen, W. A. Tolbert, and D. D. Dlott, J. Chem. Phys. 99, 4140-4151 (1993); S. Chen, W. A. Tolbert, and D. D. Dlott, J. Phys. Chem. 98, 7759-7766 (1994).

[6] P. Maffre and M. Peyrard, Phys. Rev. B 45, 9551-9561 (1992); J. Phys. - Condensed Matter 6, 4869-4878 (1994).

[7] E. Fermi, J. R. Pasta, and S. M. Ulam, in Collected Works of Enrico Fermi, Vol. II (University of Chicago Press, Chicago, 1965), pp. 978-980.

[8] G. H. Derrick, J. Math. Phys. 5, 1252-1254 (1962).

[9] G. B. Whitham, Linear and Nonlinear Waves (John Wiley \& Sons, New York, 1974).

[10] R. K. Dodd, J. C. Eilbeck, J. D. Gibbon, and H. C. Morris, Solitons and Nonlinear Wave Equations, (Academic Press, New York, 1982).

[11] Alan Newell, Solitons in Mathematics and Physics (SIAM, Philadelphia, 1985).

[12] Xidi Wang, David W. Brown, Katja Lindenberg and Bruce J. West, Phys. Rev. A 37, 3557-3566 (1988).

[13] R. Boesch and M. Peyrard, Phys. Rev. B 43, 8491-8508 (1991).

[14] H. Feddersen, in Nonlinear Coherent Structures in Physics and Biology, Lecture Notes in Physics, Vol. 393, edited by Michel Remoissenet and Michel Peyrard (Springer-Verlag, Berlin, 1991) pp. 160-167.

[15] Y. S. Kivshar and M. Peyrard, Phys. Rev. A, 46, 3198-3205 (1992).

[16] G. A. Samara and P. S. Peercy, in Solid-State Physics, Vol. 36, edited by H. Ehrenreich, F. Seitz, and D. Turnbull (Academic Press, New York, 1981), pp. 1-118.

[17] S. Califano, Lattice Dynamics of Molecular Crystals, (Springer-Verlag, New York, 1981).

[18] A. C. Newell and D. J. Kaup, Proceedings of the Royal Society A, 361, 413-446 (1978). 
[19] P. G. Drazin, Solitons (Cambridge University Press, New York, 1983).

[20] A. R. Bishop, M. G. Forest, D. W. McLaughlin, and E. A. Overman, Phys. Lett. A 144, 17-25 (1990); N. M. Ercolani, M. G. Forest, D. W. McLaughlin, and A. Sinha J. Nonlinear Science, 3, 393-426 (1993); N. M. Ercolani, D. W. McLaughlin, and H. Reitner, J. Nonlinear Science, 3, 477-539 (1993).

[21] T. C. Gard, Introduction to Stochastic Differential Equations (Marcel Dekker, New York, 1988).

[22] J. Honerkamp, Stochastic Dynamical Systems: Concepts, Numerical Methods, Data Analysis, (VCH, New York, 1993).

[23] R. W. Armstrong, W. Elban, Mat. Sci. and Eng. A 122, L1-L3 (1989); H. W. Sandusky, B. C. Glancy, D. W. Carlson, W. L. Elban, et al., J. Propulsion and Power 7, 518-525 (1991); F. A. Bandak, R. W. Armstrong, and A. S. Douglas, Phys. Rev. B 46, 3228-3235 (1992); F. A. Bandak, D. H. Tsai, R. W. Armstrong, and A. S. Douglas, Phys. Rev. B 47, 11681-11687 (1993).

[24] C. S. Coffey, J. Appl. Phys. 66, 1654-1657 (1989); 70, 4248-4254 (1991); Phys. Rev. B 49, 208-214 (1994).

[25] R. A. Marcus and Norman Sutin, Biochimica et Biophysica Acta 811, 265-322 (1985).

[26] Leon Sanche, IEEE Transactions on Electrical Insulation 28, 789-819 (1993). 